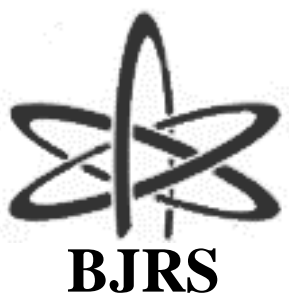

BRAZILIAN JOURNAL $\mathrm{OF}$ RADIATION SCIENCES

07-02B (2019) 01-18

\title{
A comparative study of boron transport models in NRC thermal-hydraulic code TRACE
}

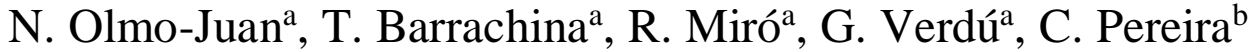 \\ ${ }^{a}$ Institute for Industrial, Radiophysical and Environmental Safety (ISIRYM), Universitat Politècnica de València, Camí \\ de Vera s/n, 46021, València, Spain \\ ${ }^{b}$ Departamento de Engenharia Nuclear, Universidade Federal de Minas Gerais, Av. Contorno, 842/9 $9^{\circ}$ andar, Centro, \\ 30110-060 Belo Horizonte, MG, Brasil \\ nioljua@iqn.upv.es,claubia@nuclear.ufmg.br
}

\begin{abstract}
Recently, the interest in the study of various types of transients involving changes in the boron concentration inside the reactor, has led to an increase in the interest of developing and studying new models and tools that allow a correct study of boron transport. Therefore, a significant variety of different boron transport models and spatial difference schemes are available in the thermal-hydraulic codes. According to this interest, in this work it will be compared the results obtained using the different boron transport models implemented in the NRC thermal-hydraulic code TRACE. To do this, a set of models has been created using the different options and configurations that could have influence in boron transport. These models allow us to reproduce a simple event of filling or emptying the boron concentration in a long pipe. Moreover, with the aim to compare the differences obtained when one-dimensional or three-dimensional components are chosen, it has modeled many different cases using only pipe components or a mix of pipe and vessel components. In addition, the influence of the void fraction in the boron transport has been studied and compared under close conditions to a BWR commercial model. A final collection of the different cases and boron transport models are compared between them and those corresponding to the analytical solution provided by the Burgers equation. From this comparison, important conclusions are drawn that will be the basis of modeling the boron transport in TRACE adequately. Keywords: boron transport, TRACE, high order schemes.
\end{abstract}




\section{INTRODUCTION}

The importance of the accuracy in the simulation of boron transport in nuclear reactor lies on the fact that boron dilution have a great importance in order to manage the reactivity control, together to the movement of the control rods, in pressurized water reactors (PWR) and to maintain the core integrity during ATWS-kind severe accidents, in boiling water reactor (BWR) in which under certain circumstances a boron injection is required.

Recently the interest in developing suitable tools that allow the reproduction of various types of transients involving the variation of the boron concentration inside the reactor [1] has led to an increase of the studies of new models and numeric schemes and its accuracy.

In first order numeric schemes the process of discretization of mass and energy equations involves the introduction of numerical diffusion that avoids performing high accuracy simulations [2]. To minimize numerical diffusion different high order schemes have been developed. Nevertheless, some second order schemes are not reliable since they can produce non-real oscillations.

In safety analyses, thermal-hydraulic calculations have to be accurate and reliable; they have to be able to reproduce real transients always with the same prediction. And in the case of transients involving a dilution or an injection of boron it is crucial to be able to simulate the boron transport within each component of the model.

Following this line of interest, the aim of this paper is to test the thermal-hydraulic code TRACE to simulate the boron transport with the different second order schemes that it has in the ßeta version. For this, a set of models in TRACE using 1D and 3D components, under single-phase and two phase thermal-hydraulic conditions, is done and the results are compared to the analytical solution provided by the Burgers equation.

The paper is organized as follows: Section 2 is devoted to explain the basis of the different schemes implemented in TRACE and the Burgers equation. Section 3 explains the models and transients used to test the different schemes. The results and discussions are in Section 4. And finally, Section 5 summarizes the main conclusions of this work. 


\section{BASICS OF THE SCHEMES ANALYZED}

\subsection{First order and second order schemes.}

In TRACE [3] there are 4 different second order schemes [4] implemented in the $\beta$ eta version for the resolution of the diffusion term of the equations: MUSCL [5], Van Leer [6], OSPRE [7] and ENO [8]. In this section, a brief review of these methods and the first order upwind method is presented to show the basics of each one of them.

The expressions are developed to the boron density calculation, which is the goal of this work. Let's start with the solute mass conservation equation:

$$
\frac{\partial\left[(1-\alpha) \rho_{\ell} C_{b}\right]}{\partial t}+\nabla\left[(1-\alpha) \rho_{\ell} C_{b} \vec{v}_{\ell}\right]=S
$$

The nomenclature used for the spatial discretization is shown in Figure 1.

Figure 1: Nomenclature used for spatial discretization

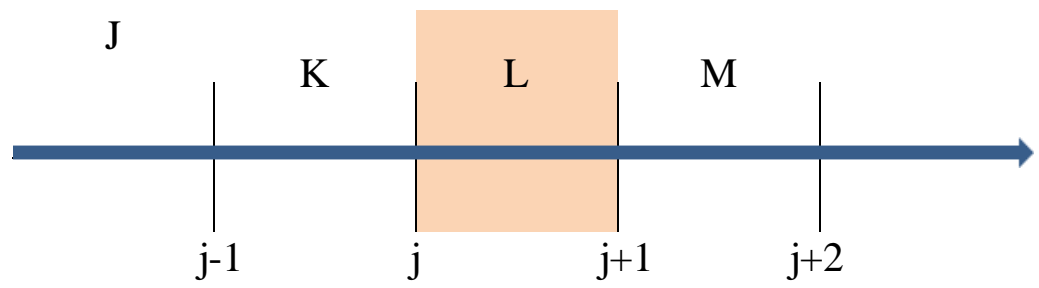

For the semi-implicit temporal integration method the equation is:

$$
\frac{\left[(1-\alpha) \rho_{\ell} C_{b}\right]_{L}^{n+1}-\left[(1-\alpha) \rho_{\ell} C_{b}\right]_{L}^{n}}{\Delta t}+\nabla_{j}\left[\left((1-\alpha) \rho_{\ell} C_{b}\right)^{n} v_{\ell}^{n+1}\right]=0
$$


Despite the fact that the first term of the equation is similar for all the schemes, the second term is defined in different way for each scheme. It should be noted that the study has not considered the possibility that the solute precipitate so it is not taken into account in equations.

In case of the first order upwind scheme we have:

$$
\frac{\rho_{b, L}^{n+1}-\rho_{b, L}^{n}}{\Delta t}+\frac{\left(\rho_{b, L}^{n} v_{\ell, j+1}^{n+1}-\rho_{b, K}^{n} v_{\ell, j}^{n+1}\right)}{\Delta x_{L}}=0
$$

where

$$
\rho_{b}=(1-\alpha) \rho_{\ell} C_{b}
$$

Otherwise the equation for the second order schemes is:

$$
\frac{\rho_{b, L}^{n+1}-\rho_{b, L}^{n}}{\Delta t}+\frac{\left(\rho_{b, L}^{n} v_{\ell, j+1}^{n+1}+\phi\left(r_{j+1}\right) \frac{\rho_{b, M}^{n}-\rho_{b, L}^{n}}{2}-\rho_{b, K}^{n} v_{\ell, j}^{n+1}+\phi\left(r_{j}\right) \frac{\rho_{b, L}^{n}-\rho_{b, K}^{n}}{2}\right)}{\Delta x_{L}}=0
$$

where $\phi\left(r_{i}\right)$ is the flux limiter with:

$$
\begin{array}{r}
r_{j+1}=\frac{\rho_{b, L}^{n}-\rho_{b, K}^{n}}{\rho_{b, M}^{n}-\rho_{b, L}^{n}} \\
r_{j}=\frac{\rho_{b, K}^{n}-\rho_{b, J}^{n}}{\rho_{b, L}^{n}-\rho_{b, K}^{n}}
\end{array}
$$

The flux limiter $\phi\left(\mathrm{r}_{\mathrm{i}}\right)$ present in equations is a nonlinear function that takes different expressions according to the chosen second order schemes. In Table 1 the flux limiter of each of the schemes analyzed are presented.

Table 1: Flux limiters

\begin{tabular}{|l|c|}
\hline \multicolumn{1}{|c|}{ Scheme } & $\phi\left(r_{i}\right)$ \\
\hline MUSCL & $\operatorname{Max}[0, \min (1.5 \mathrm{r}, 0.5(1+\mathrm{r}), 1.5]$ \\
\hline
\end{tabular}




\begin{tabular}{|l|c|}
\hline Van Leer & $(\mathrm{r}+|\mathrm{r}|) /(1+|\mathrm{r}|)$ \\
\hline ENO & $\operatorname{Max}[0, \min (\mathrm{r}, 1)]$ \\
\hline OSPRE & $1.5\left(\mathrm{r}^{2}+\mathrm{r}\right) /\left(\mathrm{r}^{2}+\mathrm{r}+1\right)$ \\
\hline
\end{tabular}

\subsection{Analytical Solution of Linearized Burgers Equation.}

The linearized Burgers equation [9] is:

$$
\frac{\partial \rho_{b}}{\partial t}+\frac{\partial\left(\rho_{b} v_{b}\right)}{\partial x}=\frac{\partial}{\partial x}\left(D \frac{\partial \rho_{b}}{\partial x}\right)+S
$$

Equation (8) has an analytical solution that allows evaluating the accuracy of the different first order and second order schemes described above. The time-dependent boundary condition is a Heaviside function, or step function, applied at the pipe inlet. The initial conditions for the analytical test are:

\section{Emptying initial conditions:}

$$
C(x, 0)= \begin{cases}C_{0}, & \text { if } x>0 \\ 0, & \text { if } x \leq 0\end{cases}
$$

The above function practically represents the transport of a boron front diluted in water assuming that the void fraction is constant in the channel. Considering that the wave moves at velocity $v=v_{f}$, no boron is generated inside the pipe $S=0$ and making the variable change $z=x-v_{f} t$ (therefore, the observer stands at the center of the wave), equation (8) can be rewritten as:

$$
\frac{\partial C}{\partial t}=D \frac{\partial^{2} C}{\partial z^{2}}
$$

Equation (9) is a diffusion equation, which has a known solution. Therefore, the exact solution of equation (9) for the case of simulation of an emptying pipe is: 


$$
C(x, t)=\frac{C_{0}}{2}\left[1-\operatorname{erf}\left(\frac{v t-x}{2 \sqrt{D t}}\right)\right]
$$

For the analogous case of filling, the initial conditions and the analytical solution would be:

$\underline{\text { Filling initial conditions: }}$

$$
\begin{array}{r}
C(x, 0)= \begin{cases}0, & \text { if } x>0 \\
C_{0}, & \text { if } x \leq 0\end{cases} \\
C(x, t)=\frac{C_{0}}{2}\left[1-\operatorname{erfc}\left(\frac{v t-x}{2 \sqrt{D t}}\right)\right]
\end{array}
$$

\section{ANALYSIS OF THE BORON TRANSPORT MODELS IN TRACE}

\subsection{Models in TRACE}

The study of the capabilities in TRACE for tracking the boron transport is based on the analysis of various simulations considering the use of $1 \mathrm{D}$ and 3D components as well as single-phase and twophase thermal-hydraulic conditions. The aim of this work is to analyze a boron injection and a dilution in all the cases considered from a steady-state condition.

The 1D model consists of a long pipe with boundary conditions at the inlet and at the outlet. Two models have been tested: horizontal and vertical pipe.

In both models, the pipe has a length of 60 meters and it is divided in 99 cells with a uniform area of $50 \mathrm{~cm} 2$. Moreover, with the aim of studying the influence of the void fraction in the boron tracking, two different thermal-hydraulic conditions have been implemented, as it is presented in table 2. Thus, in the first of them a subcooled liquid condition, at $350 \mathrm{~K}\left(76.85^{\circ} \mathrm{C}\right)$ and 43 bar, very far from its saturation temperature, is studied. On the other hand, a saturated two-phase fluid condition at 69.9 bar (closely to the pressure inside a nuclear reactor in a BWR) and a inlet void fraction of 0.5 is 
chosen to reproduce the same set of simulations. In this case, the saturation temperature is 558.882 $\mathrm{K}$.

Table 2: Thermal-hydraulic properties of the single-phase and two-phase fluid

\begin{tabular}{|c|c|}
\hline Single-phase conditions & Two-phase conditions \\
\hline Inlet boundary conditions & Inlet boundary conditions \\
\hline Velocity: $\mathrm{v}=1 \mathrm{~m} / \mathrm{s}$ & Void fraction: $\alpha=0.5$ \\
\hline Temperature: $\mathrm{T}=350 \mathrm{~K}$ & Temperature: $\mathrm{T}=558.882 \mathrm{~K}$ \\
\hline Outlet boundary conditions & Outlet boundary conditions \\
\hline Pressure: $\mathrm{P}=43 \mathrm{bar}$ & Pressure: $\mathrm{P}=69.9 \mathrm{bar}$ \\
\hline
\end{tabular}

The 3D models are based on the unique 3D component in TRACE: the vessel. Thus, it has been done a VESSEL component equivalent to the 1D component. That is, a long VESSEL with 99 axial cells and only one radial and azimuthal sectors, respectively. The boundary conditions applied are the same as the 1D models. Like in the 1D models, we have tested the horizontal and the vertical vessel in a dilution and in an injection of boron.

In the 3D models, there are PIPE components that connect the VESSEL to the boundary conditions (FILL and BREAK components). The reason for this addition is that a VESSEL component cannot be directly connected to a BREAK component, so a single PIPE of the same dimensions as the BREAK is interposed between them. On the other hand, the second PIPE must be added between FILL and VESSEL components because it was observed that boron is not transmitted from the FILL to the rest of the system if a PIPE is not used to connect it to the VESSEL. In order to avoid that the presence of this PIPE at inlet affect the study of the 3D component, the pipe length considered is 1 millimeter and the flow area is the same as the vessel component.

It should be noticed that the possibility to use a vessel component in horizontal position is not possible in TRACE so it has been done an approximation by modifying the direction of the gravitational force in order to obtain a pseudo-horizontal vessel. 


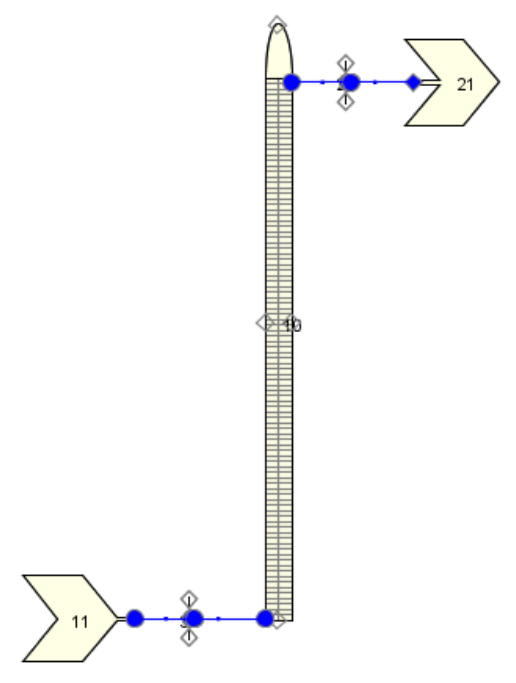

Figure 2: Model implemented in TRACE using tridimensional components

The aim of this model is to analyze if the boron tracking in the tridimensional components is done correctly, as well as to analyze the possible variations that could be generated by the different joints between components. Especially in the case where exists two-phase fluid conditions in the system. In all the models two transients are simulated. In the first one, a mass concentration of 2000 ppm of boron is injected from steady state conditions without any presence of boron. In the second transient a dilution is simulated. Starting with a mass concentration of $2000 \mathrm{ppm}$, pure water is introduced in the system in order to dilute the initial boron mass concentration.

Table 3: Description of the simulated transient

\begin{tabular}{|c|c|}
\hline Filling model & Emptying model \\
\hline$t<0 \rightarrow C_{b}=0 \mathrm{ppm}$ & $t<0 \rightarrow C_{b}=2000 \mathrm{ppm}$ \\
\hline$t=0 \rightarrow C_{b}=2000 \mathrm{ppm}$ & $t=0 \rightarrow C_{b}=0 \mathrm{ppm}$ \\
\hline
\end{tabular}

The simulated transient lasts 100 seconds and the results are shown for the center cell of the pipe (the $50^{\text {th }}$ of 99 cells) which allows evaluating the boron front at this point.

To sum up, four models in TRACE has been created in order to simulate four different cases each one. Thus, we have the following list of cases: 
Table 4: Summary of the simulations considered

\begin{tabular}{|c|c|}
\hline Models in TRACE & Different cases \\
\hline Horizontal PIPE & Single-phase filling \\
\hline Vertical PIPE & Two-phase filling \\
\hline Horizontal VESSEL & Single-phase emptying \\
\hline Vertical VESSEL & Two-phase emptying \\
\hline
\end{tabular}

\subsection{Parameters in TRACE for simulating the boron tracking}

According to the available specifications in TRACE concerning the tracking of the boron concentration, the following variables have been explored:

- NAMELIST VARIABLES

- ISOLCN: In all the cases, it is activated with a value equal to 0 . This option allows modifying the characteristic solubility of the specified solute. If " 0 " option is selected, it has been used the default solubility curve. It is noteworthy that this table only specifies the solubility of boron in water with temperature, there being no temperature values beyond $110^{\circ} \mathrm{C}$, that is, quite far from the temperature of the simulated cases. Despite of this, the low concentration of boron used $(2000 \mathrm{ppm})$ is far from the maximum solubility considered, leaving out the possibility that the solute precipitates. So that option is not really interesting in our simulations. The other options refer to the possibility of modifying the solubility curve and how to specify it as a table, function, etc.

- FRACB10: Its use refers to the calculation of the reactivity due to the variation of boron concentration in the system, and since there is no neutron process in the simulation performed, its use has been considered unnecessary.

- ISOLUT: Available from the SNAP/TRACE Model Options menu, it is also called Solute Tracking. It has three different options, "0" in case you do not want to follow the boron, "1" if you want to follow but without recalculating the density of the solvent-solute mixture and "2" for those cases where the concentration of solute could be so considerable that the density of the mixture should be recalculated. This last option 
is not available since SNAP menu. Moreover, some tests have been done trying to choose the " 2 " option, using the input file of TRACE, but it does not work with the executable used (TRACE patch 4). So, for the cases studied, the option "1" is chosen.

- MAXCONC: This option is recommended for determining a convergence limit in the calculation of the precipitated solute. Its use is only needed in case the value of the selected ISOLUT variable is greater than the unit.

- VESSEL

- ICONC: This option is specified in the menu corresponding to the component "VESSEL". It is also specified as a Solute Option and has three different options: "0" if no solute is contemplated in the liquid, " 1 " if it is contemplated, but without considering precipitation and "2", if it could be dissolved and precipitate solute. Since the boron concentration considered is clearly far of the maximum solubility, the "1" option is selected.

\section{RESULTS AND DISCUSSION}

In this section, the results obtained in the different simulations are presented. Along the various figures presented below, the evolution of the boron concentration acquired for the different models in TRACE and the numeric schemes explained in the previous sections are shown. In all the cases, this evolution tried to reproduce a step input increasing or decreasing the boron concentration.

The results obtained in the case of boron injection and dilution under thermal-hydraulic boundary conditions of single-phase liquid, are shown in Figures 3 and 4. It is easy to appreciate that no differences exist among the different configuration modelled in TRACE for the first order Upwind. Furthermore, it can be noticed that the moment, when the front evolution predicted by TRACE changes, is close to the analytical solution, but the way in wich is represented the step entrance is clearly inadequate. 
Figure 3: Results of TRACE using Upwind solution for the single-phase filling and the analytical solution

\section{FILLING}

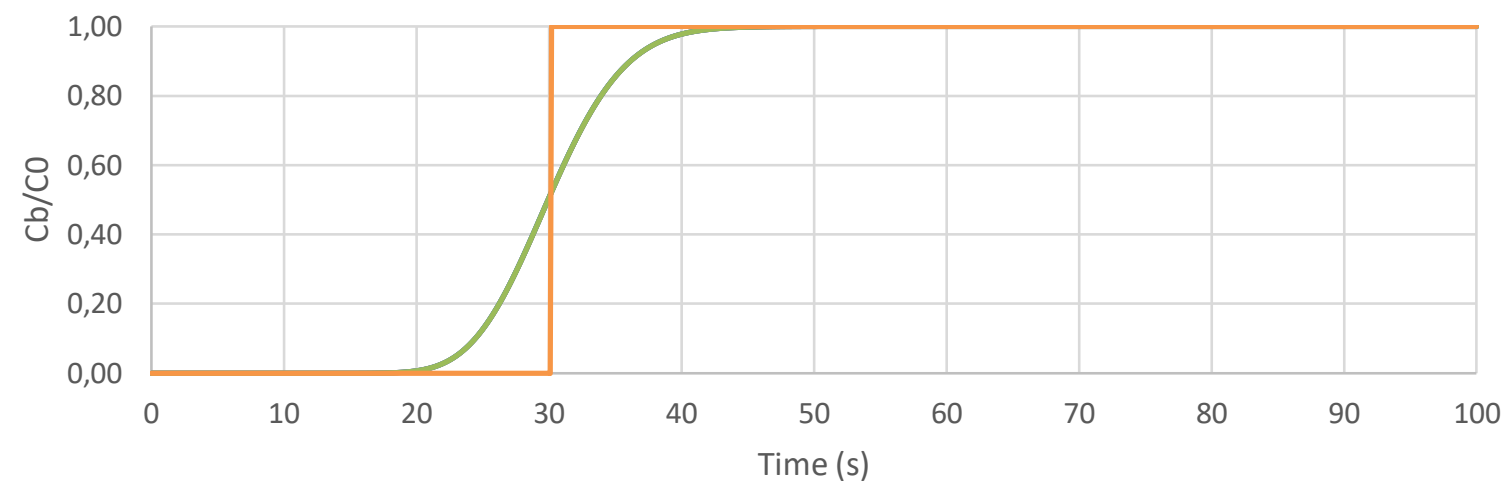

Horizontal pipe $\longrightarrow$ Vertical pipe - Horizontal vessel $—$ Vertical vessel $\longrightarrow$ ANALYTICAL

Figure 4: Results of TRACE using Upwind solution for the single-phase emptying and the analytical solution

\section{EMPTYING}

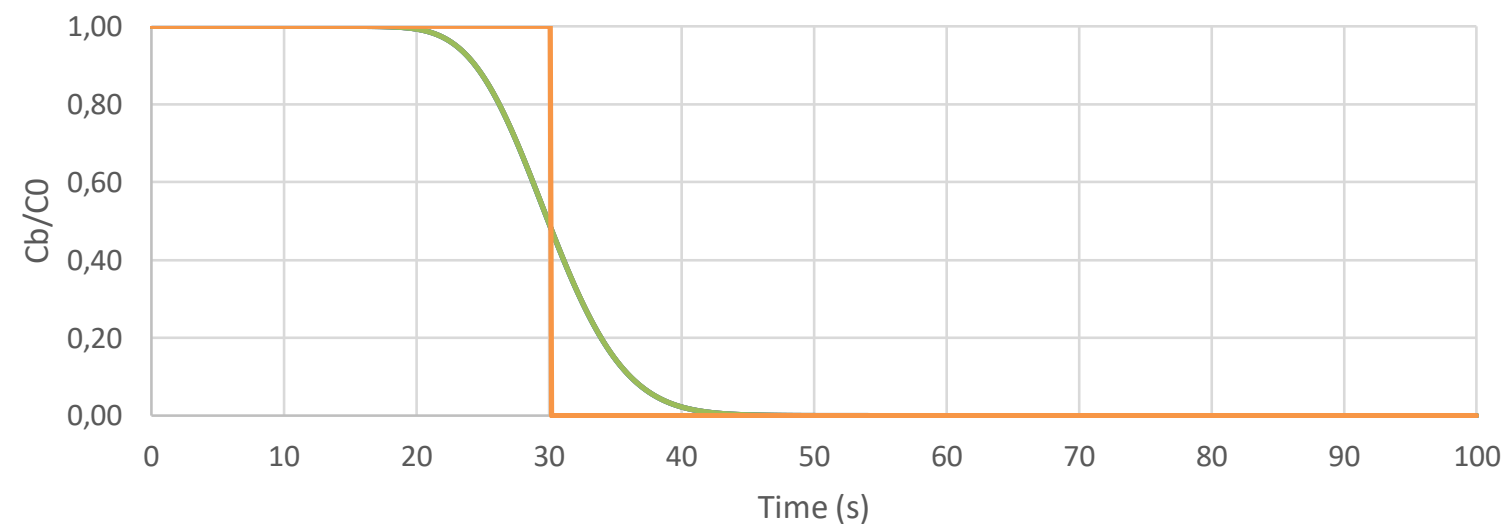

- Horizontal pipe $\longrightarrow$ Vertical pipe $\longrightarrow$ Horizontal vessel $—$ Vertical vessel $\longrightarrow$ ANALYTICAL 
With the aim to obtain a more reliable reproduction of the step input variation, it has been modified the numeric schemes chosen in TRACE simulations. Thus, first order upwind scheme, which is originally working by defect, has been replaced by the second order schemes available in TRACE. The modification of this parameter is done since Namelist Variable menu, selecting different values for the SPACEDIF parameter. Moreover, if a second order scheme is chosen, the numeric method defined in parameter NOSETS must be semi-implicit.

In figures 5 and 6 are shown the results of TRACE simulations using different numeric schemes in the case of a vertical vessel. As it was presented in the previous figures 3 and 4 , the boron evolution under single-phase boundary conditions is similar in all the cases, so a vertical vessel has been selected for this comparison.

Five different numeric schemes have been compared and the results in both cases, filling and emptying, shows that the second order schemes present a more adequate step reproduction regarding the analytical solution, being the results of second order MUSCL scheme the most suitable. Besides, should be noted that the results obtained for Second-order ENO and OSPRE method are the same and the correspondent lines are overlapped.

Figure 5: Results of TRACE using different numeric schemes for the single-phase filling and the analytical solution

\section{FILLING}

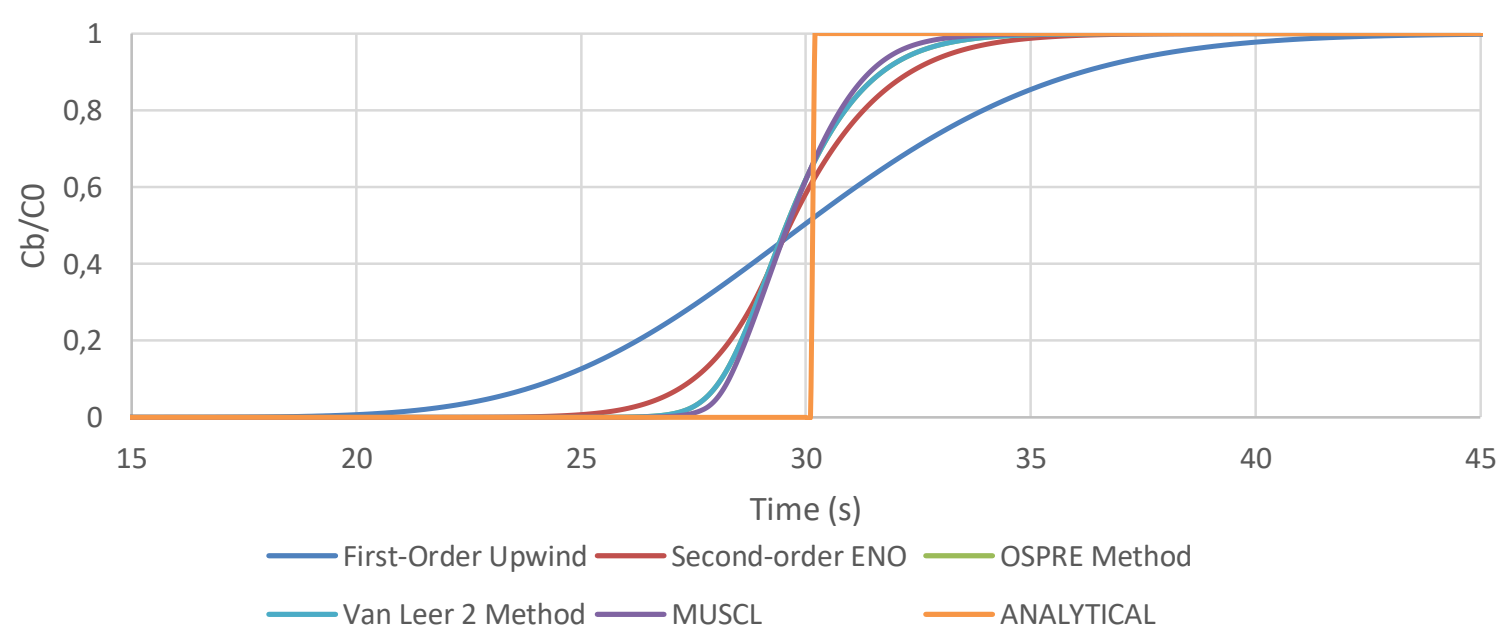


Figure 6: Results of TRACE using different numeric schemes for the single-phase emptying and the analytical solution

\section{EMPTYING}

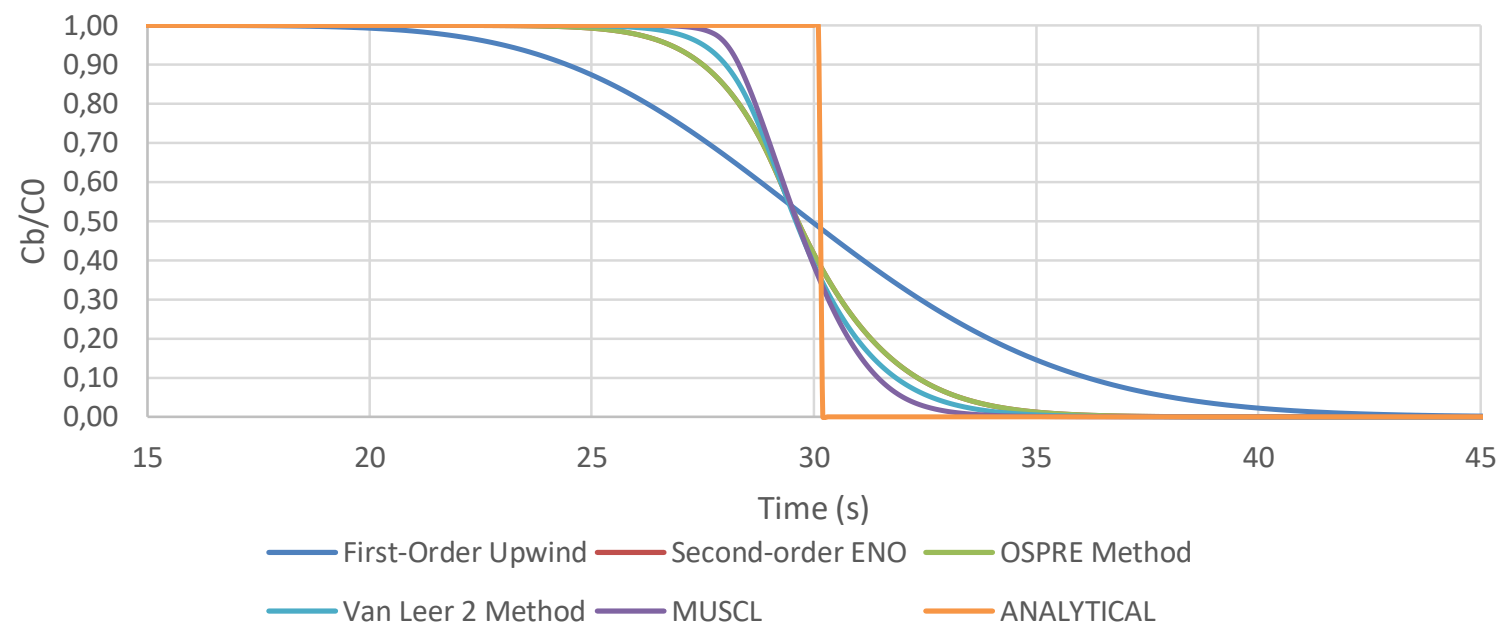

The results for boron injection and dilution under two-phase boundary condition are presented in figures 7 and 8 . Contrarily to the equivalent results for the single-phase boundary condition simulation, it has been obtained big differences between some of the cases simulated. Thereby, the horizontal cases of pipe and vessel components shows a clear delay to the correspondent to their vertical options, which are quite close but not identical. This delay and the big delay that can be appreciated with respect to the previous cases are due to the existence of various void fraction in the steady state conditions. It should be also reminded that the analytical solution is only valid for the single-phase boundary conditions. 
Figure 7: Results of TRACE using Upwind solution for the two-phase filling and the analytical solution

\section{FILLING}

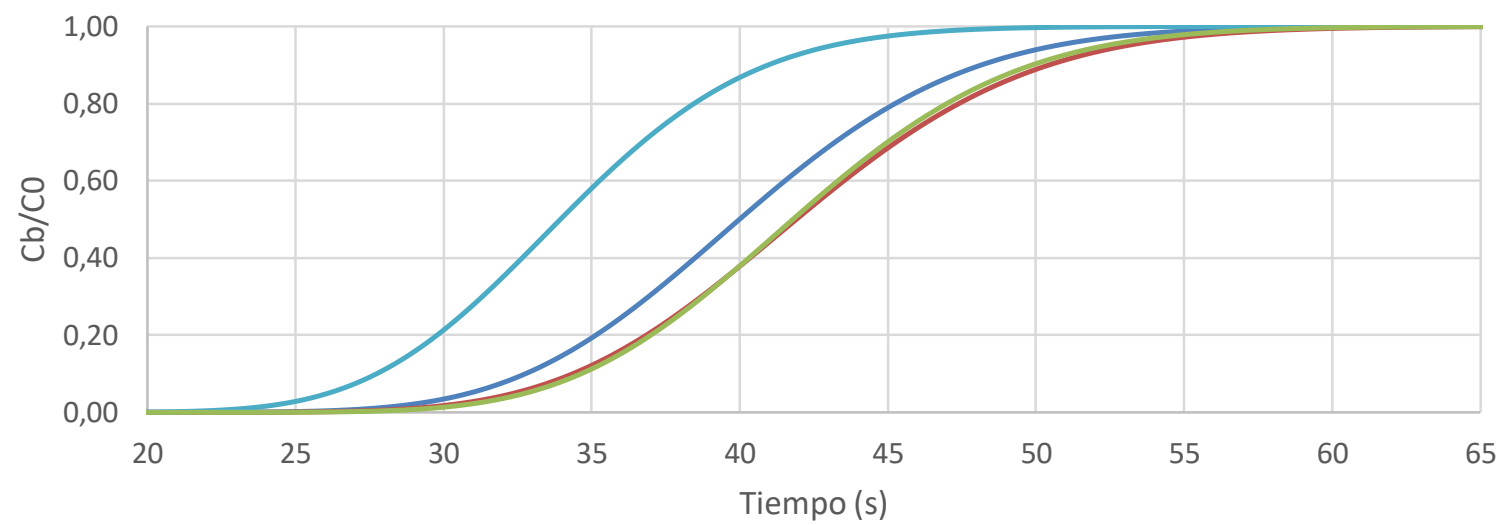

- Horizontal pipe $\quad$ Vertical pipe $\quad$ Horizontal vessel $\quad$ Vertical vessel

Figure 8: Results of TRACE using Upwind solution for the two-phase emptying and the analytical solution

\section{EMPTYING}

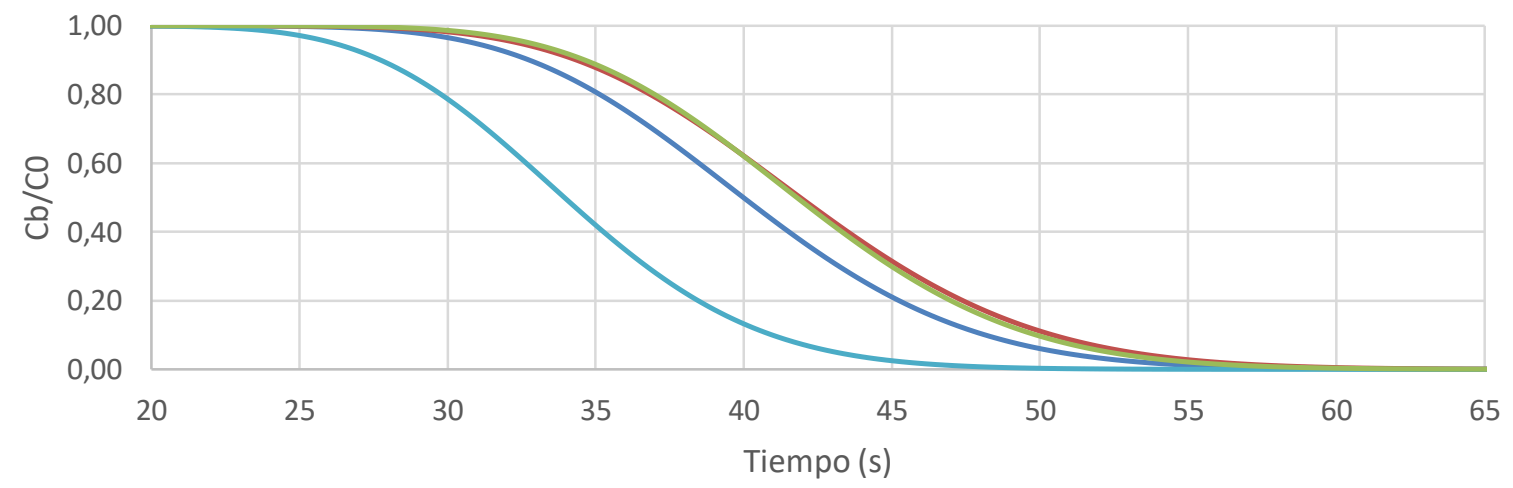

- Horizontal pipe $\quad$ Vertical pipe $\quad$ Horizontal vessel $\quad$ Vertical vessel 
The values of the converged void fraction, presented in table 4, shown a great similarity between vertical pipe and vessel, and a small variance in the case of the horizontal pipe. However, big differences exist in the case of the horizontal vessel. The fact of not using a real horizontal vessel, which is not implemented in TRACE, is considered an explanation for that big variance. As it was explained previously, we are considering a pseudo-horizontal vessel which is a vertical component with the gravitational force direction turned.

Table 4: Summary of the converged void fractions for the different models

\begin{tabular}{|c|c|}
\hline Models in TRACE & Converged void fraction \\
\hline Horizontal PIPE & 0.3208 \\
\hline Vertical PIPE & 0.3025 \\
\hline Horizontal VESSEL & 0.4265 \\
\hline Vertical VESSEL & 0.3029 \\
\hline
\end{tabular}

Finally, a comparison between the different numeric schemes in two-phase boundary conditions is shown in figures 9 and 10. The component chosen for this study is the vertical vessel that allows us to study a 3D component with a void fraction close to the correspondent to the 1D component. Furthermore, the analytical model used to solve the Burgers equation has suffered a modification for taking into account that the boron is only carried in the liquid phase, i.e. the boron is not dissolved in the vapour. For this reason, the area and the velocity of the analytical model has been modified for considering the converged values of the liquid phase as the input in the analytical model solved by Burgers equation.. As in the previous case, the second order MUSCL scheme is the option that allows a better reproduction of the step input boron evolution, Second-order ENO and OSPRE method show now differences and the evolution predicted by the first order Upwind method is clearly unsatisfying. 
Figure 9: Results of TRACE using different numeric schemes for the two-phase filling and the approximation of the analytical solution

FILLING

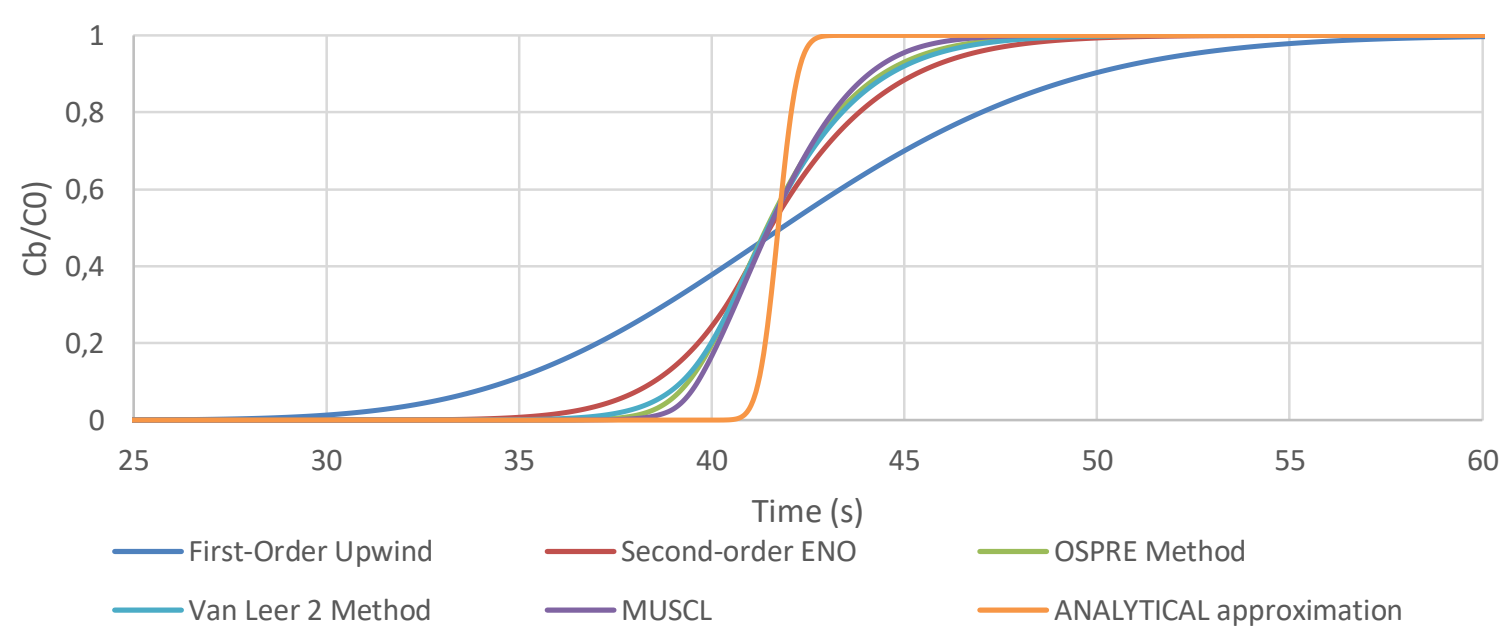

Figure 10: Results of TRACE using different numeric schemes for the two-phase emptying and the approximation of the analytical solution

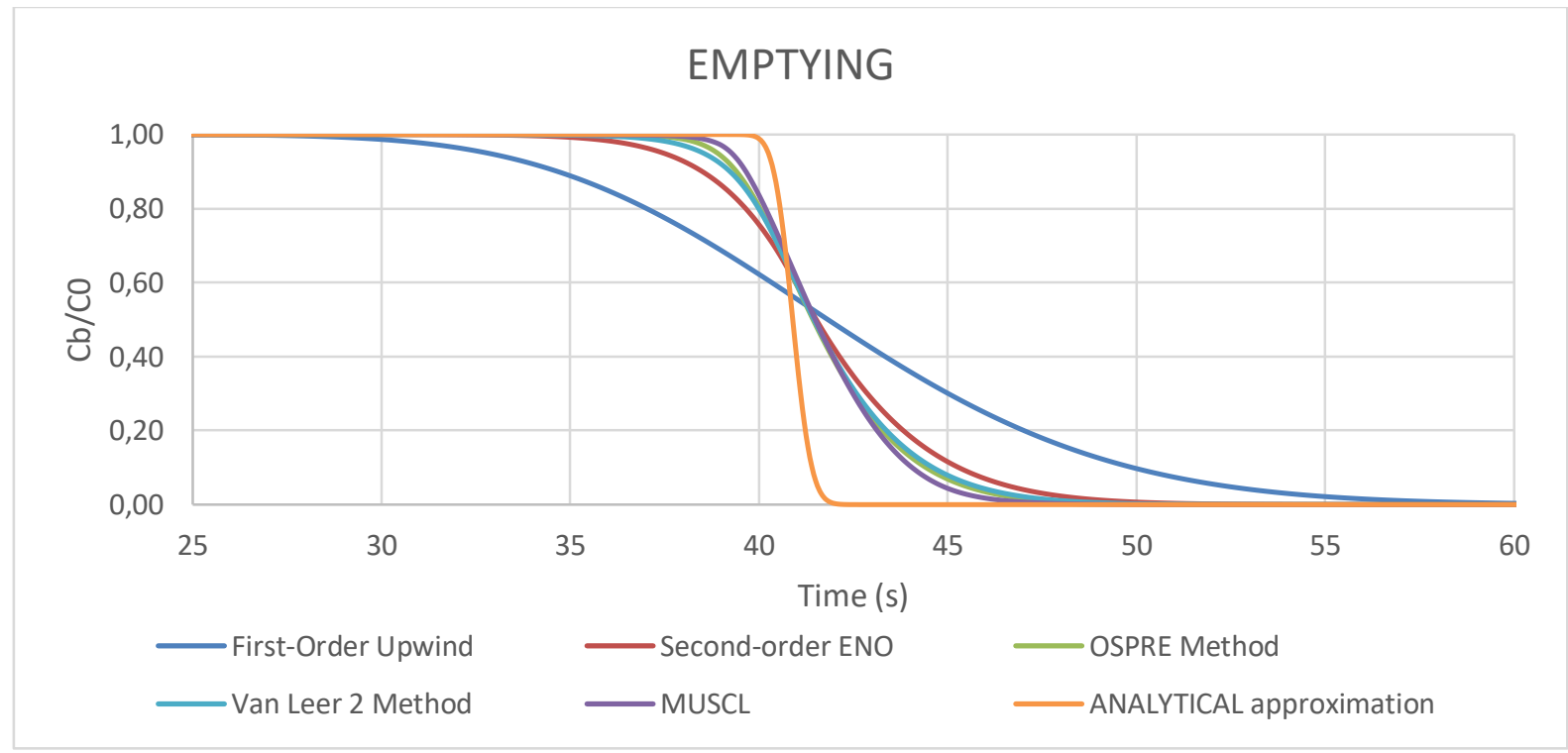




\section{CONCLUSIONS}

In the present work a set of models in TRACE have been modelled allowing to study the behavior of horizontal and vertical 1D and 3D components, under single-phase or two-phase thermal-hydraulic conditions and comparing the influence of the different numeric schemes, first and second order, available in TRACE.

The conclusions of this study is that first order Upwind scheme is not reliable for the transmission of a step input, like the most of events involving boron injection or dilution. Instead of this method, it is more suitable to use the second order MUSCL scheme, which is the closer scheme to the analytical solution, or its approximation, provided by the Burgers equation.

It has also been tested the boron evolution under two-phase boundary condition allowing to understand better the influence of the void fraction in the observed delay.

\section{REFERENCES}

[1] BARRACHINA T., SOLER A., OLMO-JUAN N., MIRÓ R., VERDÚ G. J., CONCEJAL A. Development of a high order boron transport scheme, In: JOINT INTERNATIONAL CONFERENCE ON MATHEMATICS AND COMPUTATION (M\&C), SUPERCOMPUTING IN NUCLEAR APPLICATIONS (SNA) AND THE MONTE CARLO (MC) METHOD, 2015, Nashville,TN, USA. CD-ROM, American Nuclear Society, LaGrange Park, IL (2015)

[2] OLMO-JUAN N., BARRACHINA T.M., MIRÓ R., QUEROL A., VERDÚ G.J., PEREIRA C. A comparative study of boron transport in thermal-hydraulic codes TRACE vs TRAC-BF1 and TRACE vs RELAP5, In: 17TH INTERNATIONAL TOPICAL MEETING ON NUCLEAR REACTOR THERMAL HYDRAULICS, 2017, Xi' an, China.

[3] U. S. Nuclear Regulatory Commission, TRACE V5.0 - Theory Manual, Washington, DC 20555 (2012). 
[4] WANG, D.; MAHAFFY, JH.; STAUDENMEIER, J.; THURSTON, CG., Implementation and assessment of high-resolution numerical methods in TRACE, Nuclear Engineering anwhatd Design, 263, pp 327-341, 2013.

[5] VAN LEER, B.; Towards the ultimate conservative difference scheme. V. A second-order sequel to Godunov's method. Journal of Computational Physics 32, 101-136.

[6] VAN LEER, B.; Towards the ultimate conservative difference scheme II. Monotonicity and conservation combined in a second order scheme. Journal of Computational Physics 14 (4), 361$370,1974$.

[7] N.P. WATERSON, H. DECONINCK. A unified approach to the design and application of bounded higher-order convection schemes. In: 9TH INTERNATIONAL CONFERENCE ON NUMERICAL METHODS IN LAMINAR AND TURBULENT FLOW, 1995, Taylor, Durbetaki (Eds.), Proceedings of the Ninth International Conference on Numerical Methods in Laminar and Turbulent Flow, Atlanta, July, 1995. Pineridge Press, Swansea, p. 203.

[8] SHU, C.W.; OSHER, S.; Efficient implementation of essentially non-oscillatory shock capturing scheme II. Journal of Computational Physics 83, 32-78, 1989.

[9] Burgers, J. M. The Nonlinear Diffusion Equation. D. Reidel Publishing Company, Dordrecht, Holland (1974). 